This item was submitted to Loughborough's Research Repository by the author.

Items in Figshare are protected by copyright, with all rights reserved, unless otherwise indicated.

\title{
Probabilistic impacts of climate change on flood frequency using response surfaces I: England and Wales
}

PLEASE CITE THE PUBLISHED VERSION

http://dx.doi.org/10.1007/s10113-013-0563-y

\section{PUBLISHER}

(c) Springer-Verlag Berlin Heidelberg

\section{VERSION}

AM (Accepted Manuscript)

\section{PUBLISHER STATEMENT}

This work is made available according to the conditions of the Creative Commons Attribution-NonCommercialNoDerivatives 4.0 International (CC BY-NC-ND 4.0) licence. Full details of this licence are available at: https://creativecommons.org/licenses/by-nc-nd/4.0/

\section{LICENCE}

CC BY-NC-ND 4.0

\section{REPOSITORY RECORD}

Kay, Alison L., Sue M. Crooks, Helen N. Davies, Christel Prudhomme, and Nick S. Reynard. 2019. "Probabilistic Impacts of Climate Change on Flood Frequency Using Response Surfaces I: England and Wales”. figshare. https://hdl.handle.net/2134/21120. 


\section{Probabilistic impacts of climate change on flood frequency using response surfaces I: England and Wales}

Kay, A.L., Crooks, S.M., Davies, H.N., Prudhomme, C. and Reynard, N.S.

Centre for Ecology and Hydrology, Maclean Building, Crowmarsh Gifford, Wallingford, Oxfordshire, OX10 8BB, UK

\section{Abstract}

The impacts of climate change on hydrology are an important focus of research around the world, but use of large ensembles to drive impact models is not necessarily straightforward and has to be re-done when new projections are released. Here an alternative sensitivity framework approach is demonstrated, using a set of typical response surfaces alongside the probabilistic UK Climate Projections (UKCP09). These projections comprise sets of 10,000 changes in a number of variables, available for 10 river-basin regions covering England and Wales. Estimates of the potential range of impacts on 20-year return period flood peaks are presented for different types of catchment in each region. Regional average impact ranges are compared for a number of time-horizons and emissions scenarios. Results show clear differences in impacts between catchments of different types and between regions. South-East England has the highest impacts with the greatest uncertainty range, while the Dee region has the lowest impacts and smallest uncertainty range. Regional differences are due to both spatial differences in projections and a differing regional balance in the number of catchments of each type. Ease of application of multiple projections is a clear advantage of this sensitivity-based approach to impact assessment, which could be extended to other regions and sectors.

\section{Keywords}

Response surfaces; climate change; floods; adaptation

\section{Introduction}

The potential impacts of climate change on the water cycle are of growing concern around the world, particularly in terms of flood and drought frequency (IPCC 2007; Section 3.4.3). Data from Global or Regional Climate Models (GCMs/RCMs) are often used to drive hydrological models, to assess impacts. Examples include; Lehner et al. (2006), who looked at both floods and droughts in Europe using the WaterGAP model with data from two GCMs; Dankers and Feyen (2009), who investigated flooding in Europe using the LISFLOOD model with data from two RCMs nested in two GCMs; and Bell et al. (2012), who investigated flooding in the Thames Basin, UK, using the G2G model with data from an 11-member RCM ensemble.

The production of new, ever-larger, climate model ensembles makes the impact modelling process more onerous (Fronzek et al. 2010). The probabilistic UK Climate Projections 2009 (UKCP09, Murphy et al. 2009) are a case-in-point, as they consist of sets of 10,000 (equally likely) changes in several variables, for a 
number of emissions scenarios and time-horizons, on a $25 \mathrm{~km}$ grid or for regions across the UK. For winter daily mean temperatures in Britain, the projections for the 2080s under the Medium emissions scenario suggest a median $\left(50^{\text {th }}\right.$ percentile) increase of between 2 and $3^{\circ} \mathrm{C}$, with a $10^{\text {th }}-90^{\text {th }}$ percentile range of approximately $1-4^{\circ} \mathrm{C}$. Temperature increases are slightly greater for summer than winter, and over southern regions than more northerly ones. For precipitation, the projections for the 2080s under Medium emissions suggest a median change in annual total precipitation near zero (widest $10^{\text {th }}$ $90^{\text {th }}$ percentile range $-8-7 \%$ ), but with a median winter increase of between $11 \%$ (range 1-25\%; eastern Scotland) and 23\% (range 5-54\%; south-west England) and a median summer decrease between -24\%(range -49-6\%; southwest England) and -11\% (range -28-4\%; northern Scotland). Doing an impact study for many catchments with such a large set of probabilistic climate projections is not necessarily straightforward, particularly if more than one emissions scenario and/or time-horizon is required, and will need to be redone when new projections are released.

To reduce this computational burden, a number of recent studies have approached impact assessment from the other direction, using a sensitivity framework (e.g. Wetterhall et al. 2011, Weiß 2011, Bastola et al. 2011, Fronzek et al. 2010, Prudhomme et al. 2010). This involves the definition of a regular sensitivity domain comprising a large number of plausible scenarios of climatic change. Modelling is then used to define the change in a given indicator (e.g. winter runoff) for each scenario of the sensitivity domain, producing a 'response surface'. Any given climate projection can be overlaid on the response surface and an impact estimate extracted from the nearest corresponding point of the domain. Thus impacts from a large set of projections can be estimated quickly and easily. Provided the sensitivity domain has been defined appropriately the response surfaces should remain valid for new projections.

Prudhomme et al. (2010) developed a sensitivity domain to investigate impacts of climate change on flood peaks in Britain. Prudhomme et al. (2013a) implemented this domain for 154 catchments across Britain and grouped the catchment response surfaces, leading to nine 'response types' each represented by composite (average) response surfaces. Prudhomme et al. (2013b) characterised families of response types by catchment properties, enabling estimation of type for any catchment where the necessary properties are available. Thus the impacts of multiple projections can be easily estimated even for un-modelled catchments, by using composite response surfaces for the appropriate type.

This paper

a. Develops the response type estimation method of Prudhomme et al. (2013b), to allow robust application to a large set of catchments across England and Wales.

b. Overlays the UKCP09 projections on the composite response surfaces of Prudhomme et al. (2013a), to estimate the impacts (percentage changes in flood peaks) for each response type in regions across England and Wales.

c. Combines $\mathrm{a}$ and $\mathrm{b}$ above, to produce weighted regional impacts. 
The background and impact assessment method are described in Section 2, with results in Section 3 and discussion and conclusions in Section 4. The results support regional vulnerability assessments and policy-making for adaptation under climate change. A companion paper further develops the method for Scotland (Kay et al. 2013a).

\section{Impact assessment method}

\subsection{Background}

The sensitivity domain developed by Prudhomme et al. (2010) relies on use of a single-harmonic function to represent the monthly pattern of changes in precipitation $(\mathrm{P})$ and temperature $(\mathrm{T})$, allowing the dimensionality of the domain to be greatly reduced while maintaining a seasonal variation. The function is given by

$$
X(t)=X_{\text {mean }}+A \cos [2 \pi(t-\Phi) / 12]
$$

with $X(t)$ change for month $t$, harmonic mean $X_{\text {mean }}$ (mean annual change), harmonic amplitude $A$ (height of peak above mean) and harmonic phase $\Phi$ (month of peak). Prudhomme et al. (2010) analysed multiple climate model projections for Britain, to determine appropriate values/ranges for the harmonic function parameters for $P$ and $T$. For $P$, the analysis showed that the vast majority of projections had a peak change in winter, so the phase $\Phi$ was set to 1 (January). Thus the sensitivity domain involved only two dimensions of $P$ change $\left(X_{\text {mean }}\right.$ and $A$ ), each varied in $5 \%$ increments between minimum and maximum values $\left(-40 \%-60 \%\right.$ for $X_{\text {mean }} ; 0 \%-100 \%$ for $\left.A\right)$ to give 525 scenarios of $\mathrm{P}$ change. The analysis also showed no significant correlation between $\mathrm{P}$ and $\mathrm{T}$ changes, so $\mathrm{T}$ changes were treated independently and few scenarios were used as floods in Britain are much less sensitive to $T$ than $P$ change. Thus the sensitivity domain involved eight scenarios of $\mathrm{T}$ change, each with corresponding potential evaporation (PE) changes, giving a total of 4,200 scenarios when combined with the 525 scenarios of $P$ change (8x525) (Supplementary Table 1). The sensitivity framework thus consisted of applying the changes given by the sensitivity domain using delta change downscaling.

Using this sensitivity framework, 154 catchments across Britain were modelled (Prudhomme et al. 2013a). Details on the hydrological modelling, which included a temperature-dependent snowmelt module, are given by Crooks et al. (2009). For each scenario of the sensitivity domain, peaks-over-threshold were extracted from simulated flow series (using an implicit threshold to give an average of two peaks per year). A flood frequency curve was then fitted to the extracted peaks, using a Generalised Pareto Distribution, and this curve compared to a baseline curve to calculate changes in 20-year return period flood peaks (RP20). These were presented as a 2-d response surface for each catchment and each T/PE scenario, with $P$ harmonic amplitude $(A)$ on the $x-$ axis, $P$ harmonic mean $\left(X_{\text {mean }}\right)$ on the $y$-axis, and the colour of the square at each point representing the modelled change in RP20 for that scenario of $\mathrm{P}, \mathrm{T}$ and PE change. The response surfaces of the 154 catchments were grouped by similarity (using a hierarchical clustering method), forming nine response types (Prudhomme et al. 2013a). These types were approximately ordered and named by the sensitivity shown in their response surfaces: Damped-Extreme (DpE), Damped-High (DpH), Damped-Low (DpL), Neutral (Neu), Mixed (Mix), Enhanced-Low (EnL), Enhanced-Medium (EnM), Enhanced-High (EnH) and 
Sensitive (Sen). Each type was represented by a composite (average) response surface and standard deviation surface (representing the range of responses within each type) (Figure 1a). For Damped types, the response surfaces show percentage changes in flood peaks generally smaller than the corresponding $P$ changes, while flood peak changes for Enhanced types are often larger than the $P$ changes. The Neutral type has changes similar to those for $P$, while changes for Mixed and Sensitive types are more dependent on the specific seasonality and magnitude of $P$ changes.

Prudhomme et al. (2013b) characterised families of response types by catchment properties, using a decision tree - a set of binary rules (based on catchment properties) which divide a sample (set of catchments) into a number of categories (response types/families). The families were formed by merging some of the response types, to enable more robust characterisation and take some account of variation of response surfaces with $\mathrm{P}$ harmonic phase: a Neutral/Damped family was formed by merging Damped-High, Damped-Low and Neutral catchments into one group, and an Enhanced family was formed by merging Enhanced-Low, Medium and High. The Mixed and Sensitive types remained as distinct families, but the Damped-Extreme type was excluded as it only contained three catchments so could not be characterised robustly. A wide range of catchment property data were used for the 154 catchments, from the UK National River Flow Archive (NRFA; www.ceh.ac.uk/data/nrfa/) and the Flood Estimation Handbook (FEH; www.ceh.ac.uk/feh/). The resulting decision tree (bold rules in Figure 2) can be used to estimate the response type/family for any catchment where the necessary catchment properties are available. Note that each path of the decision tree is actually associated with probabilities for each response type/family; the best-estimate is simply that with the highest probability.

Although Prudhomme et al. $(2013 a, b)$ focus on RP20, the analyses were also done for flood peaks with return periods of 2-, 10- and 50-years (Reynard et al. 2009). The same response types (with similar composite response surfaces) are valid for all return periods (although a catchment is not necessarily of the same type for each) and decision trees were derived for each (although the merging of response types into families was only applied for 20- and 50-year return periods). Results here again focus on RP20, but some discussion is included for other return periods where appropriate; full details for other return periods are given by Kay et al. (2011).

\subsection{Decision tree modification}

Over 1000 catchments in England and Wales are listed in the NRFA and have the necessary catchment properties available to apply the RP20 decision tree. However, it is necessary to check the applicability of the tree, developed on a set of 154 modelled catchments, to the larger set of NRFA catchments, which is likely to cover a wider range of catchment property combinations. This section describes minor modifications to the tree, to make it more robust.

The individual ranges of relevant catchment properties are not significantly different between the two catchment sets (not shown). The exception is Mean Annual Loss (MAL), which has a much greater range in the NRFA set than the modelled set. This is not surprising since, for model calibration purposes, the 
modelled set represented relatively natural catchments where MAL is dominated by evaporative losses. In contrast, the NRFA set includes catchments with more anthropogenic water usage, including high gains and high losses. An inspection of use of the decision tree for catchments with high or low MAL indicated that it should still be applicable.

Although the ranges of individual catchment properties do not appear to affect use of the tree for the larger set of catchments, there are combinations of properties that may do so. That is, based on hydrological understanding of the response types, there are implicit thresholds used in the tree, due to combinations of catchment property values in the modelled set, that need to be made explicit for use with the NRFA set. These involve the combinations SAAR and BHP, and MAL and BHP (see Figure 2 for definitions).

The modelled set contains no catchments with both high SAAR and high BHP. Consequently, the original decision tree assigns Neutral/Damped or Mixed response types to catchments with high SAAR $(>969.5 \mathrm{~mm})$, regardless of their BHP (bold rules in Figure 2). However, it was felt that BHP should be the dominant factor in these cases, thus the first rule of the original tree was modified (italics in Figure 2) to include a check on BHP as well as SAAR, using the BHP threshold from further down the original tree. Similar modifications were made to the original trees for all four return periods (Kay et al. 2011).

The modelled set contains few catchments with low SAAR, high MAL and low BHP. Consequently, Path 6 of the original decision tree assigns the Sensitive response type to catchments with low SAAR and high MAL, regardless of BHP (bold rules in Figure 2). However, there are many NRFA catchments that satisfy these two criteria, and they are unlikely to all be Sensitive; it was felt that this type should be reserved for catchments which also have high BHP, as in the modelled set. Thus the second rule in the first branch of the original tree was modified (italics in Figure 2) to include a check on BHP as well as MAL, again using the BHP threshold from further down the original tree. Similar modifications were made to the original trees for 10 - and 50-year return periods (Kay et al. 2011).

The decision trees with the above modifications (Figure 2) were considered suitable for application to the full set of NRFA catchments in England and Wales (Section 3.1).

\subsection{Use of UKCP09 projections}

UKCP09 provides probabilistic projections, termed Sampled Data, consisting of 10,000 sets of changes in a number of climate variables (Murphy et al. 2009). These are available as monthly changes from the baseline time-slice (19611990 ) to a number of future 30-year time-slices under three emissions scenarios, on an approximately $25 \mathrm{~km} \times 25 \mathrm{~km}$ grid over the UK or for 23 riverbasin regions. The latter are used here as they are consistent across any river catchment; the grid data are not spatially coherent so cannot provide spatial averages or different inputs to different parts of a catchment. There are 10 UKCP09 river-basin regions covering (the majority of) England and Wales: North-West England, Northumbria, Dee, Humber, West Wales, Anglian, Severn, Thames, South-East England, South-West England (Figure 4 top-left). Two 
further regions (Solway and Tweed) cover small parts of England but are mainly in Scotland, so are covered in the companion paper. Most of the results here are for the 2080s (2070-2099) time-horizon under Medium emissions (equivalent to A1B; IPCC 2000), although a comparison of impact ranges for alternative time-horizons and emissions is presented later.

An example showing a set of UKCP09 projections for a river-basin region overlaid on a composite response surface is presented in Figure 1b. To do this overlaying, a single-harmonic function (Section 2.1) is fitted to each of the 10,000 sets of monthly $P$ changes for the region. Two $P$ harmonic parameters (mean and amplitude) determine the position of each projection on the sensitivity domain. [See Supplementary Section 2 for a discussion of the appropriateness of the sensitivity domain simplifications for the UKCP09 projections.] Plots of the $P$ harmonic mean versus amplitude for each riverbasin region (Figure 3 ) show that the distribution of the 10,000 projections on the domain differs between regions; as well as their ranges (and medians) varying, the correlation between the two parameters also varies. Four regions have positive correlations; North-West England (+0.21), West Wales $(+0.10)$, South-West England (+0.08) and South-East England (+0.09). Two regions show little correlation; Severn $(-0.02)$ and Northumbria $(-0.04)$. The remaining four regions have negative correlations; Dee (-0.40), Humber (-0.22), Anglian ($0.16)$ and Thames $(-0.17)$. This correlation is important as the response surfaces change fastest when both harmonic mean and amplitude increase (i.e. from bottom-left to top-right of the sensitivity domain; Figure 1), so a region with a positive correlation between the two parameters (given the same ranges and medians) would have a greater impact range than a region with a negative correlation, while impacts for a region with little correlation are likely to lie somewhere in between.

In terms of harmonic mean and amplitude ranges and correlation, the Dee region's projections are more like those for easterly regions than for the westerly regions surrounding it (Figure 4 top-left), perhaps because of the Dee's location in the lee of the Welsh hills.

Only the UKCP09 projections of monthly $\mathrm{P}$ changes are used here; variations in $T$ changes are much less important for changes in flood peaks in Britain (Prudhomme et al. 2013a), so the composite response surfaces (Figure 1a) incorporate all eight T/PE scenarios together. The standard deviation surfaces then include the (small) additional uncertainty from use of composite surfaces averaged over T/PE scenarios, as well as covering the uncertainty due to the range of possible catchment responses of a given type (see Section 2.4).

\subsection{Uncertainty}

Compared to a standard top-down impact assessment (i.e. modelling impacts directly using climate projections), there are two main additional sources of uncertainty when estimating impacts using climate projections with composite response surfaces. These are;

1) representation of a catchment response surface by a composite response surface, and

2) the assumptions and simplifications necessary to develop the sensitivity framework approach. 
The methods used to allow for these two sources of uncertainty are described below. Other potential sources of uncertainty are discussed in Section 4 .

The first additional source of uncertainty can be quantified using the standard deviation (sd) surfaces, alongside the composite response surfaces (Figure 1). For each response type, as well as using the $\mathrm{P}$ harmonic mean and amplitude to extract the central-estimate of the impact from the composite response surface, they are also used to extract, from the sd surface, an estimate of the sd corresponding to that central-estimate. Adding \pm 2 sd to the central-estimate accounts for around $95 \%$ of the range (assuming $\sim$ normal distributions).

For the second additional source of uncertainty, Kay et al. (2013b) present an assessment focussing on nine catchments; one to represent each response type. The results showed different levels of uncertainty for different catchments. For example, the Neutral, Damped-Low and Enhanced-Low catchments had quite low levels of uncertainty, while the Damped-High and Mixed catchments had greater uncertainty and the Sensitive catchment had the greatest uncertainty. These differences were considered compatible with the underlying climatological and hydrological differences between response types (i.e. how and why they differ in response to changes in climatic inputs). This link between response type and level of uncertainty enables values to be derived for the correction of mean bias in the impacts extracted from response surfaces. Thus, for the four response types/families characterised by the decision tree here, the corrections are taken as: Neutral/Damped 7\%; Mixed 11\%; Enhanced 9\%; Sensitive $20 \%$. These values can be added to the impacts extracted from the response surfaces, to give more robust impact estimates. Expert judgement was exercised in the derivation of these correction values, particularly for the merged families, to allow for the different balance of uncertainties affecting each type.

\section{Results}

\subsection{Response type estimation for NRFA catchments}

The decision tree (Figure 2) is used to estimate the response type/family for 1,120 NRFA catchments in the 10 river-basin regions over England and Wales. These are summarised in Figure 4, which shows that areas in the north/west have greater homogeneity of response (predominantly Neutral/Damped) compared to areas in the south/east. This is due to higher precipitation in the north/west than the south/east, which is the dominant factor affecting catchment response in the tree. In the south/east, lower rainfall means that heterogeneity of soils/geology has more influence: Most of the UK's major aquifers are located in the south/east, and catchments containing significant areas of high permeability have Enhanced or Sensitive responses, whereas nearby catchments of predominantly lower permeability can have Mixed or Neutral/Damped responses.

\subsection{Response-type impacts}

As described in Section 2.3, the UKCP09 projections for each river-basin region (Figure 3 ) are overlaid on the composite response surfaces for each response type (Figure 1a top). An estimate of the impact of each projection is then taken from that of the nearest point of the sensitivity domain. The 10,000 projections 
per region thus become 10,000 impacts per response type per region, plotted as box-plots in Figure 5. This clearly shows the differences between response types, with the lowest median $\left(50^{\text {th }}\right.$ percentile) impact generally for DampedExtreme and the highest median impact generally for Enhanced-High. Although the size of the $25^{\text {th }}-75^{\text {th }}$ percentile range generally increases monotonically with the response type 'ordering' (Section 2.1), there is not a corresponding monotonic increase in the median impact. This is because the response type ordering concentrated on the width of the $0 \%-90 \%$ impact band on the response surfaces, but the shape of this band also varies, non-monotonically (Figure 1a top). The effect is clearest for Sensitive, which has a much lower median impact than that for Enhanced-High despite having a narrower band. The slope of the band, as the P harmonic amplitude increases, is much shallower for Sensitive than for Enhanced-High, so many projections result in lesser impacts from the Sensitive surface than the Enhanced-High one. There are similar, less pronounced, differences for Neutral and Mixed, and EnhancedLow and Enhanced-Medium. It should be noted that the box-plots in Figure 5 do not include bias correction values (Section 2.4); these could also affect the ordering.

\subsection{Weighted regional impacts}

The numbers of NRFA catchments of each response type/family in each region (Figure 4 top-right) are used to weight the response-type impact ranges (Section 3.2), to produce regional average impact ranges (right-most box-plots in Figure 5). Following Prudhomme et al. (2013b), in order to minimise possible underestimation of impacts, the range for the Neutral response type is used with the number of Neutral/Damped catchments, and the range for the EnhancedHigh response type is used with the number of Enhanced catchments. The resulting regional average impact ranges could be considered to represent a central-estimate of the average impact range for a catchment in the region, taking account of the range of UKCP09 projections and the range of response types in the region.

Figure 6 compares the regional average impact ranges for each region after bias correction values have been added for each response type/family (Section 2.4). Accounting for the bias, due to simplifications necessary for the sensitivity approach, provides a more robust central-estimate of the impact range. The regional average impact ranges show that the South-East England region has the highest impacts, with a median RP20 change of around $35 \%$ and by far the largest difference between the $10^{\text {th }}$ and $90^{\text {th }}$ percentile impacts $(\sim 9 \%-80 \%)$. The Dee region has the lowest impacts; it is the only region with a median RP20 change of less than $20 \%$ and has the smallest $10^{\text {th }}-90^{\text {th }}$ percentile range $(\sim 6 \%-$ $31 \%)$. The Northumbria and Humber regions, in north-eastern England, also have relatively low median impacts (close to $20 \%$ ) and relatively small $10^{\text {th }}-90^{\text {th }}$ percentile ranges. So the impacts for the Dee region are more like those for north-easterly regions than for the westerly regions surrounding it (as noted for the projections; Section 2.3). The other six regions have median impacts similar to each other, between about $23 \%$ and $28 \%$, with the more southerly regions generally having wider $10^{\text {th }}-90^{\text {th }}$ percentile ranges than more northerly regions.

Also shown in Figure 6 are the alternative ranges when \pm 2 sd is added to the central-estimate impact for each projection (Section 2.4). This allows for the 
uncertainty from using composite response surfaces to represent what is actually a range of possible catchment responses classified as the same response type. There is greater uncertainty from this source for some regions (e.g. Thames and South-East England) than others (e.g. North-West England and West Wales).

The regional differences in the median impact and in the range of uncertainty are a result of both spatial differences in the UKCP09 projections (Figure 3), and a differing regional balance in the number of NRFA catchments of each type (Figure 4), given the differences between the composite response surfaces (and sd surfaces) of each type (Figure 1). For example, South-East England has the greatest range of $P$ harmonic amplitudes and the highest median $P$ harmonic amplitude (Figure 3), and the highest proportion of Enhanced and Sensitive catchments (63\%; Figure 4), thus it has the highest median impact and the largest range of uncertainty (both from the 10,000 UKCP09 projections and from the sd ranges; Figure 6). Northumbria has the (equal) smallest range of $\mathrm{P}$ harmonic amplitudes and the lowest median $\mathrm{P}$ harmonic amplitude (Figure 3 ), but does not have the lowest median impact or the smallest range of uncertainty because it still has a relatively high proportion of Enhanced catchments (31\%; Figure 4$)$. The Dee region has the lowest median impact and the smallest range of uncertainty from the 10,000 UKCP09 scenarios, because it has the (equal) smallest range of $P$ harmonic amplitudes and a negative correlation between $\mathrm{P}$ harmonic amplitude and mean (Figure 3), and a relatively low proportion of Enhanced and Sensitive catchments (13\%; Figure 4). However, the Dee does not have the smallest uncertainty from the sd ranges as it does not have the lowest proportion of Enhanced and Sensitive catchments; North-West England has only $7 \%$ whilst West Wales has only $2 \%$ and these regions have the smallest uncertainty from the sd ranges.

\subsection{Regional impacts for alternative UKCP09 projections}

Results so far have used projections for the 2080s under Medium emissions. Here, comparisons are presented using Medium emissions for three timehorizons (2020s; 2050s; 2080s), and for three emissions scenarios (Low - B1; Medium - A1B; High - A1F1) for the 2080s time-horizon. To simplify the comparison, three summary numbers are chosen to illustrate the range of uncertainty. The lower end of the uncertainty range is represented by the $25^{\text {th }}$ percentile selected from the central-estimate-2sd; the middle of the uncertainty range is represented by the $50^{\text {th }}$ percentile selected from the central-estimate; the upper end of the uncertainty range is represented by the $75^{\text {th }}$ percentile selected from the central-estimate+2sd. In this way, all of the main sources of uncertainty (UKCP09 climate modelling, use of composite response surfaces, and the sensitivity framework method) are included in the lower and upper ends of the range. Maps of these summary numbers (Figure 7) show the variation in impacts for different regions, with a north/west to south/east split being evident in many of them. The first three rows of maps show the clear increase in impacts with time-horizon (from 2020s to 2080s under Medium emissions). The last three rows of maps show the variation with emissions for the 2080s, with impacts under High emissions clearly greater than for Low emissions. The impacts under Low emissions for the 2080s (row 4) are similar to those under Medium emissions for the 2050s (row 2). 


\section{Discussion and conclusions}

The sensitivity framework method provides a powerful tool enabling rapid assessment of climate change impacts (Prudhomme et al. 2010). Nine typical response types were identified (Prudhomme et al. 2013a), represented by composite response surfaces which can be combined with multiple climate projections to estimate impacts on flood peaks in Britain. Here, for 20-year return period flood peaks, the probabilistic UKCP09 projections for 10 riverbasin regions across England and Wales have been used to produce probabilistic response-type impacts. Furthermore, the decision trees of Prudhomme et al. (2013b) have been modified and applied, to estimate the response type of 1,120 NRFA catchments in the 10 regions. This information has been used to estimate regional impact ranges, by weighting response-type impact ranges.

The results show that different regions have quite different impacts, both in terms of the median impact and the range of uncertainty. This information supports updated policy on flood risk management for England, which now provides regional recommendations for climate change allowances (Environment Agency 2011) rather than the national guidelines for a $20 \%$ allowance provided previously (Defra 2006). The Welsh Government are currently working with the Environment Agency to produce revised regional guidance for Wales.

Weighting response-type impacts to make regional impacts is based only on the set of NRFA catchments in each river-basin region. It is possible that this set may not give a true representation of the distribution of response types (e.g. there may be more gauges in some areas than others, potentially skewing the distribution of response types). Future work will try to replace Mean Annual Loss in the decision tree with alternative standardised and nationally available loss estimates, enabling response type estimation even for ungauged catchments. The proportion of river points of each type in each region would then be more representative of the region as a whole, as would the resulting regional impacts. Information on the level of confidence in the best-estimate response type for each catchment (Prudhomme et al. 2013b), or on the probabilities for each response type, could also be used to adjust the weighting scheme. However, Prudhomme et al. (2013b) discuss the limited suitability of the method for highly urbanised catchments or those with high attenuation of flows due to reservoirs and lakes. Such limitations should be borne in mind.

Being able to estimate the response type for any catchment in England and Wales, gauged or ungauged, would also enable better use of the response-type impacts themselves, to obtain more catchment-specific estimates of the potential impacts than is provided by regional impacts. The range for some response types in some regions is quite different to the regional average (Figure 5). For instance, the West Wales region is dominated by Neutral catchments (Figure 4), so the regional impact range is basically that for the Neutral type (Figure 5). The use of the regional impact range to represent all catchments in the region would mean that the impact for the small number of Enhanced catchments in the region would be under-estimated, and the impact for the small number of Mixed catchments in the region would be over-estimated. Prudhomme et al. (2013b) present an example vulnerability assessment using 
GCM projections for two (geographically close) catchments in central England, illustrating their differing vulnerability to the impacts of climate change on flooding due to differing sensitivity.

Uncertainty bias correction values (Section 2.4) have been included in some of the regional impact ranges presented here, but the uncertainty assessment of Kay et al. (2013b) suggested that there was greater bias for larger catchments. Only $2.6 \%$ of the 1,120 NRFA catchments in the 10 river-basin regions over England and Wales have an area $>2,000 \mathrm{~km}^{2}$, but it could be that impacts for these catchments would be greater than suggested here.

The UKCP09 Sampled Data were designed to cover a wide range of the uncertainty in climate change projections (Murphy et al. 2009) but they are provided in the form of change factors. Using delta change downscaling with those factors limits the range of uncertainty in the impact since the factors are applied to a fixed baseline time-series (Kay and Jones 2012). The uncertainty bias correction values allow for differences in the medians using the sensitivity framework compared to time-series (Kay et al. 2013b), but the expanded range that is likely to result from use of time-series methods is not included. Neither is hydrological model parameter or structural uncertainty, although these are generally smaller than climate modelling uncertainty (e.g. Chen et al. 2011, Gosling et al. 2011, Kay et al. 2009, Thompson et al. 2013). Similarly, uncertainty in flood frequency curve fitting is not included, but likely to have a relatively small effect on the percentage changes derived, at least for lower return periods. Kay et al. (2013b) provide a wider discussion of choices within sensitivity-based approaches, and the potential influence of these on results.

It is important to remember that the UKCP09 probabilistic projections, and impacts resulting from them, are conditional on the models, methods and data used to build them (Murphy et al. 2009, Kay and Jones 2012). New sets of projections could thus be very different. The sensitivity framework method provides a relatively easy way of assessing whether new projections are likely to lead to impacts much different from existing ones. Fitting harmonic functions to the $\mathrm{P}$ changes from any new projections, and plotting harmonic mean versus amplitude (like Figure 3), would give a good indication of potential differences between sets. However, the $P$ harmonic phases from new projections should be checked, given the January phase assumption for the existing response surfaces. The T changes should also be checked, to make sure they are not too different to the eight T scenarios of Prudhomme et al. (2010). The sensitivity framework method also provides a useful tool to quickly assess the relative severity of potential impacts for a set of catchments, as the impacts for some response types are greater than others. Such a sensitivity-based approach could be extended to other regions and sectors. However, as discussed by Prudhomme et al. (2013b) and Kay et al. (2013b), use of the method should not be considered as a complete replacement for a full site-specific impact assessment, particularly for highly vulnerable situations.

\section{Acknowledgements}

This research was supported by the Department for Environment Food and Rural Affairs (Defra) (project number FD2648) with additional contribution from the NERC-CEH Water science programme. The UK Climate Projections data have been made available by the Department for 
Environment, Food and Rural Affairs (Defra) and Department for Energy and Climate Change (DECC) under licence from the Met Office, Newcastle University, University of East Anglia and Proudman Oceanographic Laboratory. These organisations accept no responsibility for any inaccuracies or omissions in the data, nor for any loss or damage directly or indirectly caused to any person or body by reason of, or arising out of, any use of this data.

\section{References}

Bastola S, Murphy C, Sweeney J (2011) The sensitivity of fluvial flood risk in Irish catchments to the range of IPCC AR4 climate change scenarios. Science of the Total Environment, 409, 54035415.

Bell VA, Kay AL, Cole SJ, Jones RG, Moore RJ, Reynard NS (2012) How might climate change affect river flows across the Thames Basin? An area-wide analysis using the UKCP09 Regional Climate Model ensemble. Journal of Hydrology, 442-443, 89-104, doi: 10.1016/j.jhydrol.2012.04.001.

Chen J, Brissette FP, Poulin A, Leconte (2011) Overall uncertainty study of the hydrological impacts of climate change for a Canadian watershed. Water Resources Research, 47, W12509, doi: 10.1029/2011WR010602

Crooks SM, Kay AL, Reynard NS (2009) Regionalised impacts of climate change on flood flows: hydrological models, catchments and calibration. Report to Department for Environment, Food and Rural Affairs, FD2020 project milestone, CEH Wallingford, November 2009, 59pp.

Dankers R, Feyen L (2009) Flood hazard in Europe in an ensemble of regional climate scenarios. Journal of Geophysical Research, 114, D16108, doi:10.1029/2008JD011523.

Defra (2006) FCDPAG3 Economic Appraisal: Supplementary Note to Operating Authorities Climate Change Impacts, October 2006, Department for Environment, Food and Rural Affairs, London, UK.

Environment Agency (2011) Adapting to Climate Change: Advice for Flood and Coastal Erosion Risk Management Authorities. Environment Agency, September 2011, 29pp.

Fronzek S, Carter TR, Räisänen J, Ruokolainen L, Luoto M (2010) Applying probabilistic projections of climate change with impact models: a case study for sub-arctic palsa mires in Fennoscandia. Climatic Change, 99, 515-534.

Gosling SN, Taylor RG, Arnell NW, Todd MC (2011) A comparative analysis of projected impacts of climate change on river runoff from global and catchment-scale hydrological models. Hydrology and Earth System Sciences, 15, 279-294.

Lehner B, Döll P, Alcamo J, Henrichs T, Kaspar F (2006) Estimating the impact of global change on flood and drought risks in Europe: A continental, integrated analysis. Climatic Change, 75, 273-299.

IPCC (2000) Special report on emissions scenarios (SRES): A special report of Working Group III of the Intergovernmental Panel on Climate Change. Cambridge University Press, Cambridge, UK, 599pp.

IPCC (2007) Climate Change 2007: Impacts, adaptation and vulnerability. Contribution of Working Group II and III to the Fourth Assessment Report of the Intergovernmental Panel on Climate Change. M.L. Parry, O.F. Canziani, J.P. Palutikof, P.J. van der Linden and C.E. Hanson (eds). Cambridge University Press, Cambridge, UK, and New York, USA, 841pp.

Kay AL, Crooks S, Davies HN, Prudhomme C, Reynard NS (2011) Practicalities for implementing regionalised allowances for climate change on flood flows. Report to Department for Environment, Food and Rural Affairs, Technical Report FD2648, CEH Wallingford, May 2011, 209pp. 
Kay, A.L., Crooks, S.M., Davies, H.N. and Reynard, N.S. (2013a). Probabilistic impacts of climate change on flood frequency using response surfaces. II: Scotland. Regional Environmental Change, submitted.

Kay AL, Crooks SM, Reynard NS (2013b) Using response surfaces to estimate impacts of climate change on flood frequency: assessment of uncertainty. Hydrological Processes, doi:10.1002/hyp.10000.

Kay AL, Davies HN, Bell VA, Jones RG (2009) Comparison of uncertainty sources for climate change impacts: flood frequency in England. Climatic Change, 92(1-2), 41-63, doi: 10.1007/s10584-008-9471-4.

Kay AL, Jones RG (2012) Comparison of the use of alternative UKCP09 products for modelling the impacts of climate change on flood frequency. Climatic Change, 114, 211-230, doi: 10.1007/s10584-011-0395-z.

Murphy JM, Sexton DMH, Jenkins GJ, Booth BBB, Brown CC, Clark RT, Collins M, Harris GR, Kendon EJ, Betts RA, Brown SJ, Humphrey KA, McCarthy MP, McDonald RE, Stephens A, Wallace C, Warren R, Wilby R, Wood RA. (2009) UK Climate Projections Science Report: Climate change projections. Met Office Hadley Centre, Exeter, UK.

Prudhomme C, Crooks S, Kay AL, Reynard NS (2013a) Climate change and river flooding: Part 1 Classifying the sensitivity of British catchments. Climatic Change, 119(3-4), 933-948, doi: 10.1007/s10584-013-0748-X.

Prudhomme C, Kay AL, Crooks S, Reynard NS (2013b) Climate change and river flooding: Part 2 Sensitivity characterisation for British catchments and example vulnerability assessments. Climatic Change, 119(3-4), 949-964, doi: 10.1007/s10584-013-0726-3.

Prudhomme C, Wilby RL, Crooks S, Kay AL, Reynard NS (2010) Scenario-neutral approach to climate change impact studies: Application to flood risk. Journal of Hydrology, 390, 198-209.

Reynard NS, Crooks S, Kay AL, Prudhomme C (2009) Regionalised impacts of climate change on flood flows. Report to Department for Environment, Food and Rural Affairs, Technical Report FD2020, CEH Wallingford, November 2009, 113pp.

Thompson JR, Green AJ, Kingston DG, Gosling SN (2013) Assessment of uncertainty in river flow projections for the Mekong River using multiple GCMs and hydrological models. Journal of Hydrology, 486, 1-30.

Weiß M (2011) Future water availability in selected European catchments: a probabilistic assessment of seasonal flows under the IPCC A1B emission scenario using response surfaces. Natural Hazards and Earth System Sciences, 11, 2163-2171.

Wetterhall F, Graham LP, Andréasson J, Rosberg J, Yang W (2011) Using ensemble climate projections to assess probabilistic hydrological change in the Nordic region. Natural Hazards and Earth System Sciences, 11, 2295-2306. 


\section{Figures}
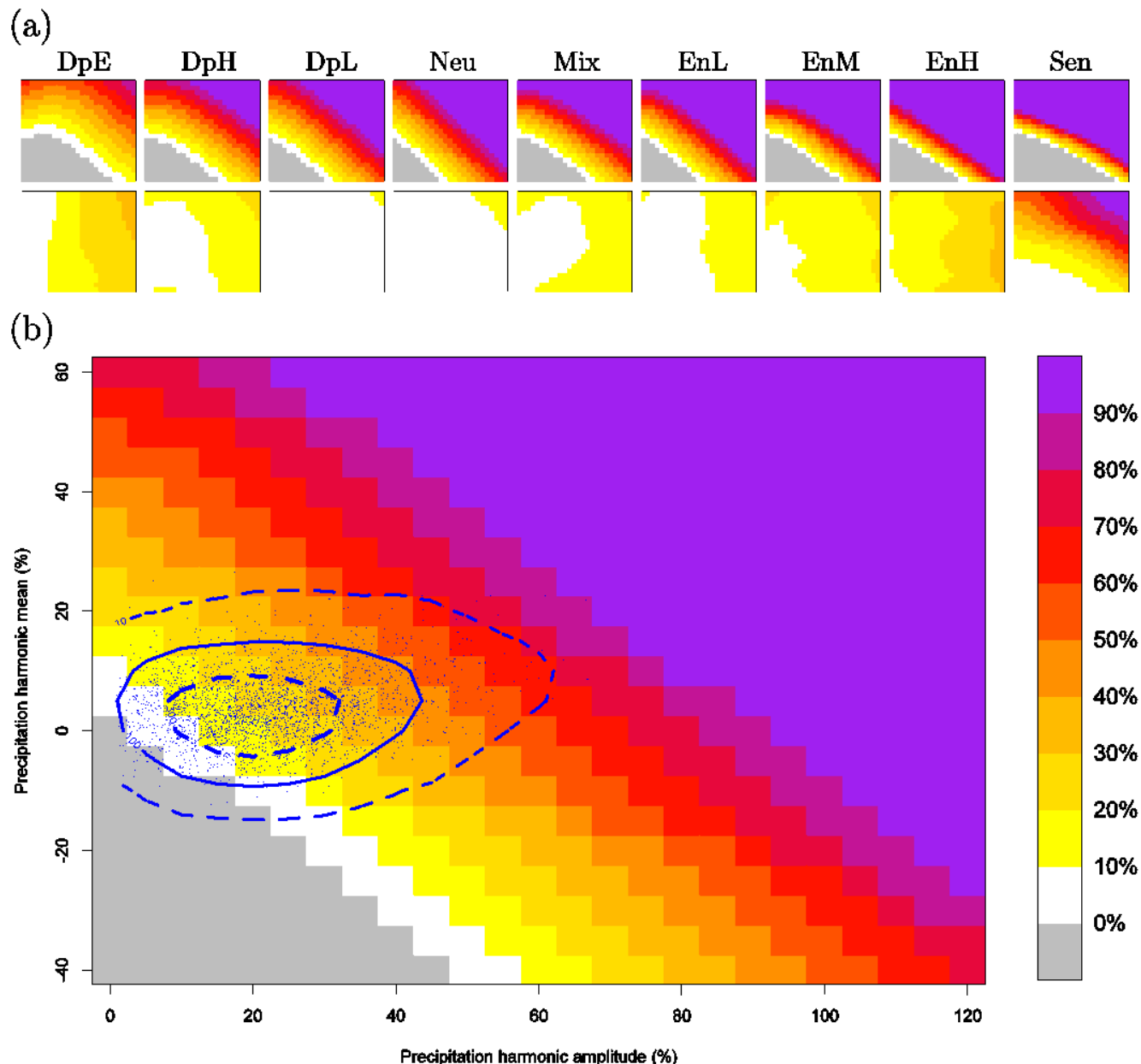

Figure 1 (a) Composite response surfaces (first row) and standard deviation surfaces (second row) for each response type, for RP20 percentage changes (see colour key). (b) Composite response surface (Neutral) overlaid with UKCP09 projections for North-West England (blue dots). Contours delineate densities of 10,100 and 300 projections per $5 \% \times 5 \%$ sensitivity domain square. 


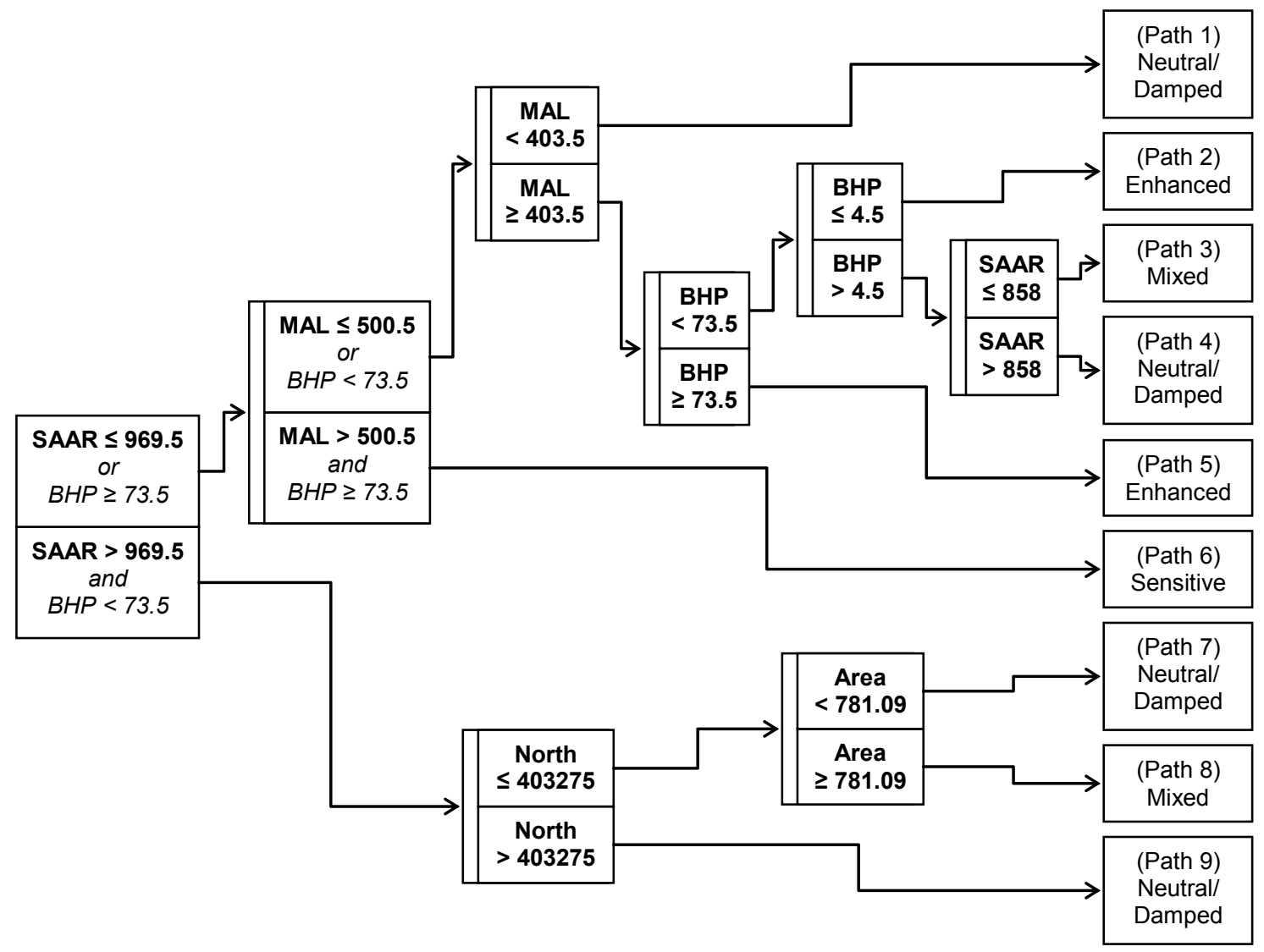

Key for catchment properties:

SAAR - Standard Average Annual Rainfall for 1961-90 (mm)

North - northing of catchment outlet (6-figure GB national grid reference)

Area - catchment area $\left(\mathrm{km}^{2}\right)$

MAL - Mean Annual Loss, the difference between catchment mean annual rainfall and runoff ( $\mathrm{mm}$ ) BHP - percentage of catchment underlain by high permeability bedrock (\%)

Figure 2 Final decision tree for England and Wales, for response type/family estimation for RP20 changes. Original rules in bold, with modifications in italics. The final column gives the path number and the best-estimate of the response type/family for each path. 

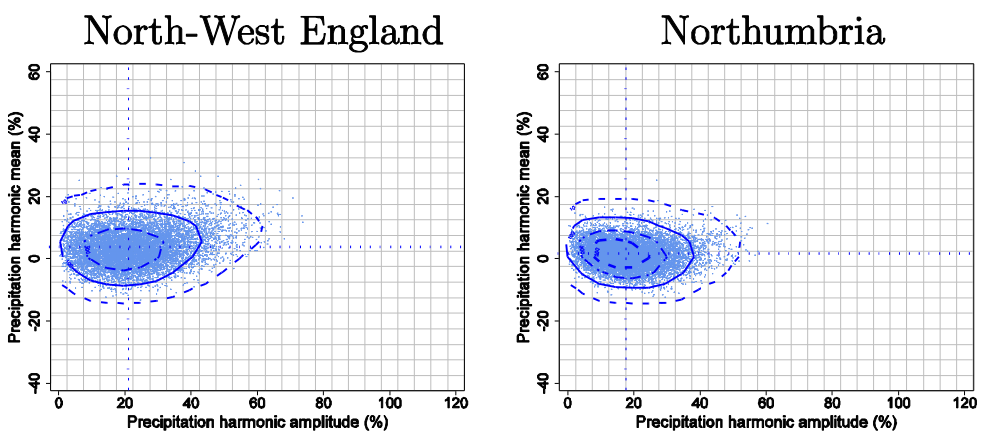

Dee

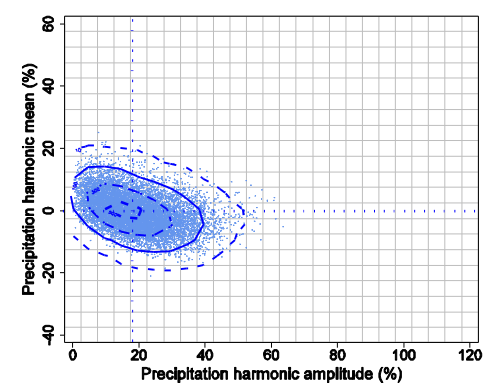

Humber

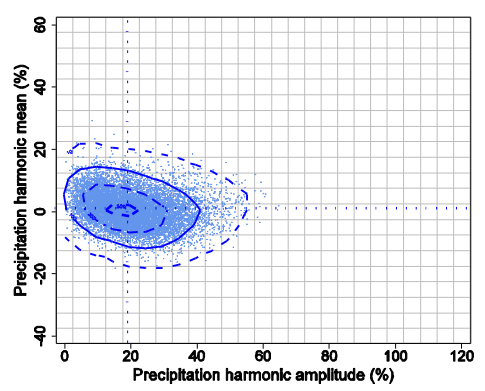

West Wales

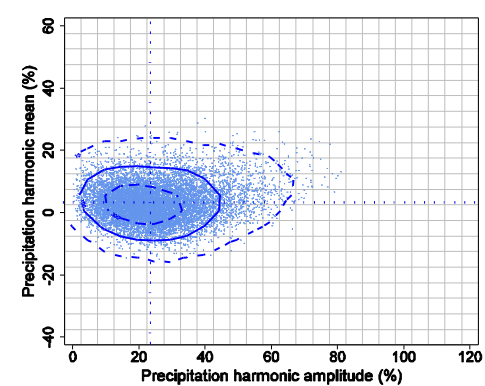

Anglian

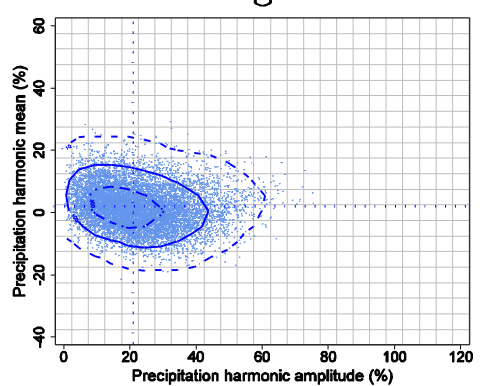

Severn

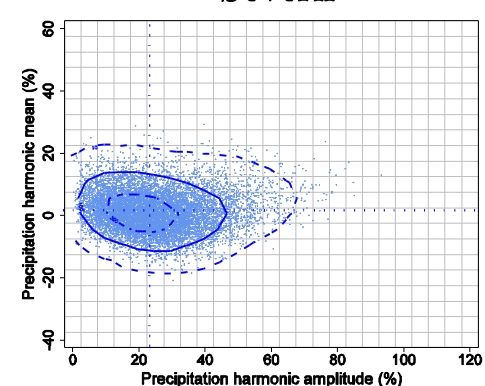

Thames

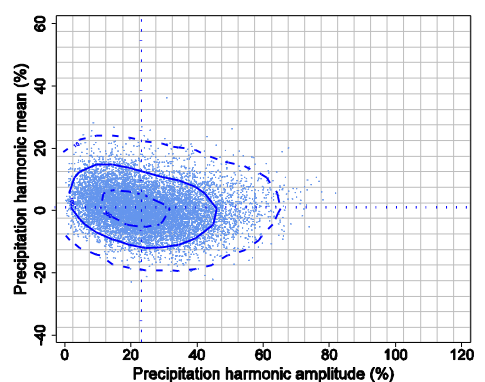

South-West England
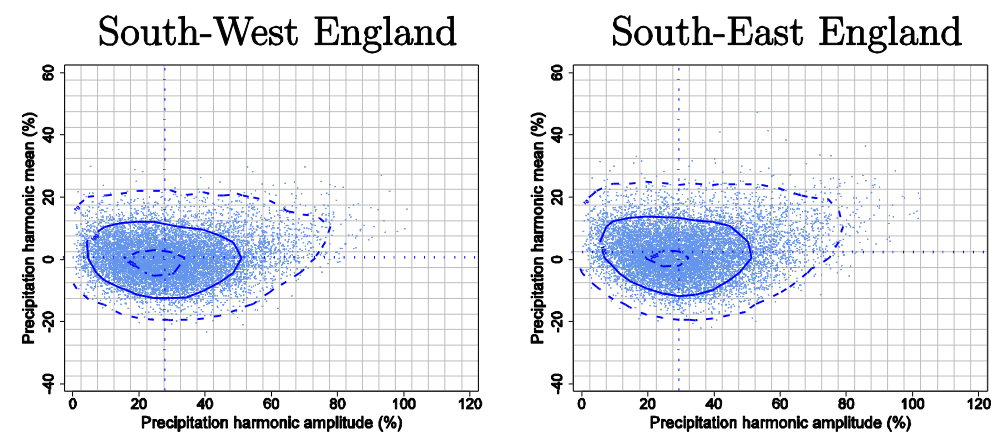

Figure $3 \mathbf{P}$ harmonic mean versus amplitude on the sensitivity domain grid, for the 10,000 UKCP09 projections (2080s Medium) for each riverbasin region in England and Wales (dots). Contours delineate densities of $10,100,300$ and (possibly) 500 projections per $5 \% \times 5 \%$ sensitivity domain square. Dotted horizontal and vertical lines indicate the median harmonic mean and amplitude respectively. 


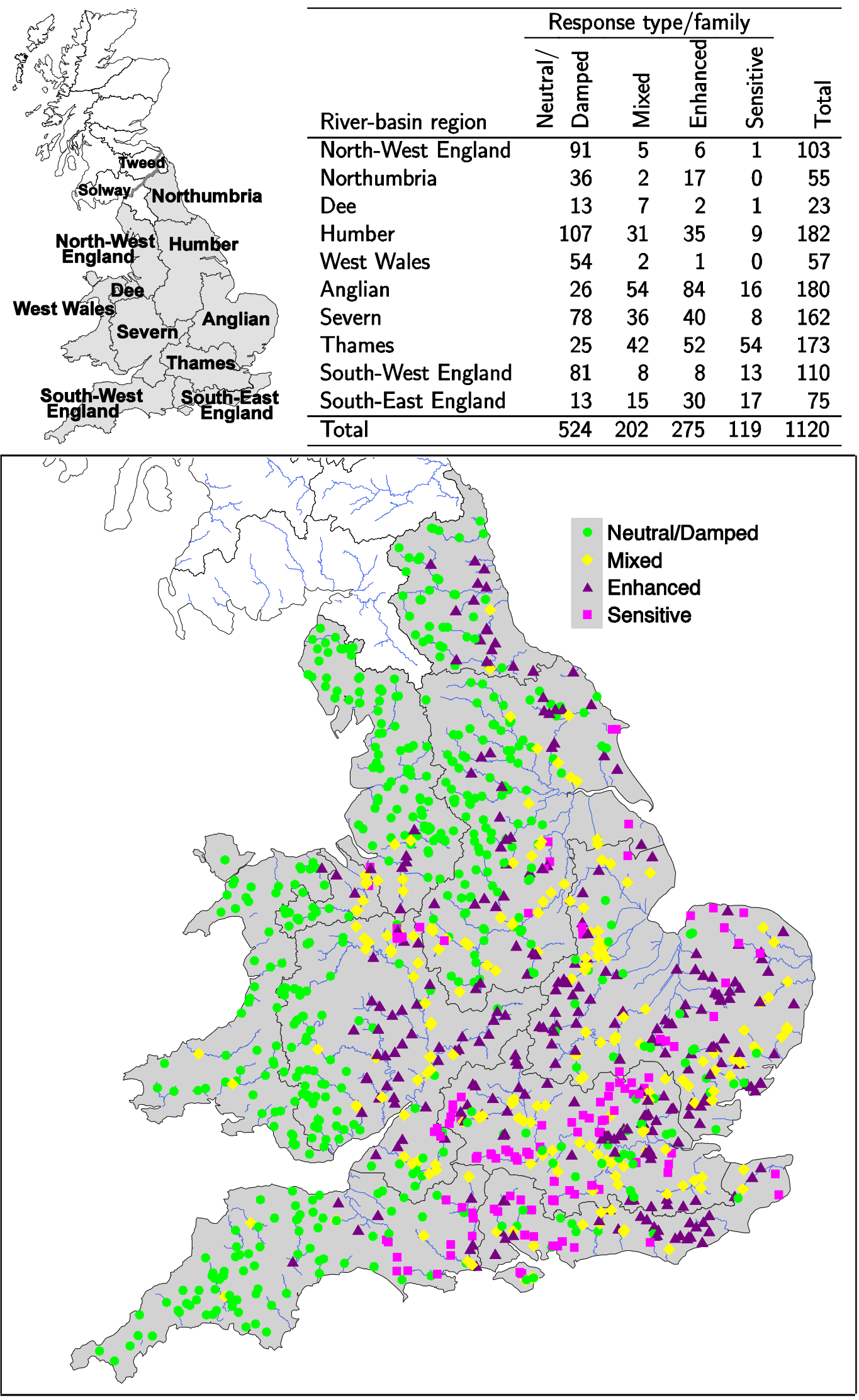

Figure 4 Summary of the estimated response type/family for each NRFA catchment in 10 river-basin regions over England and Wales. Region names are in the top-left map, with the England/Scotland border (thick grey line). 

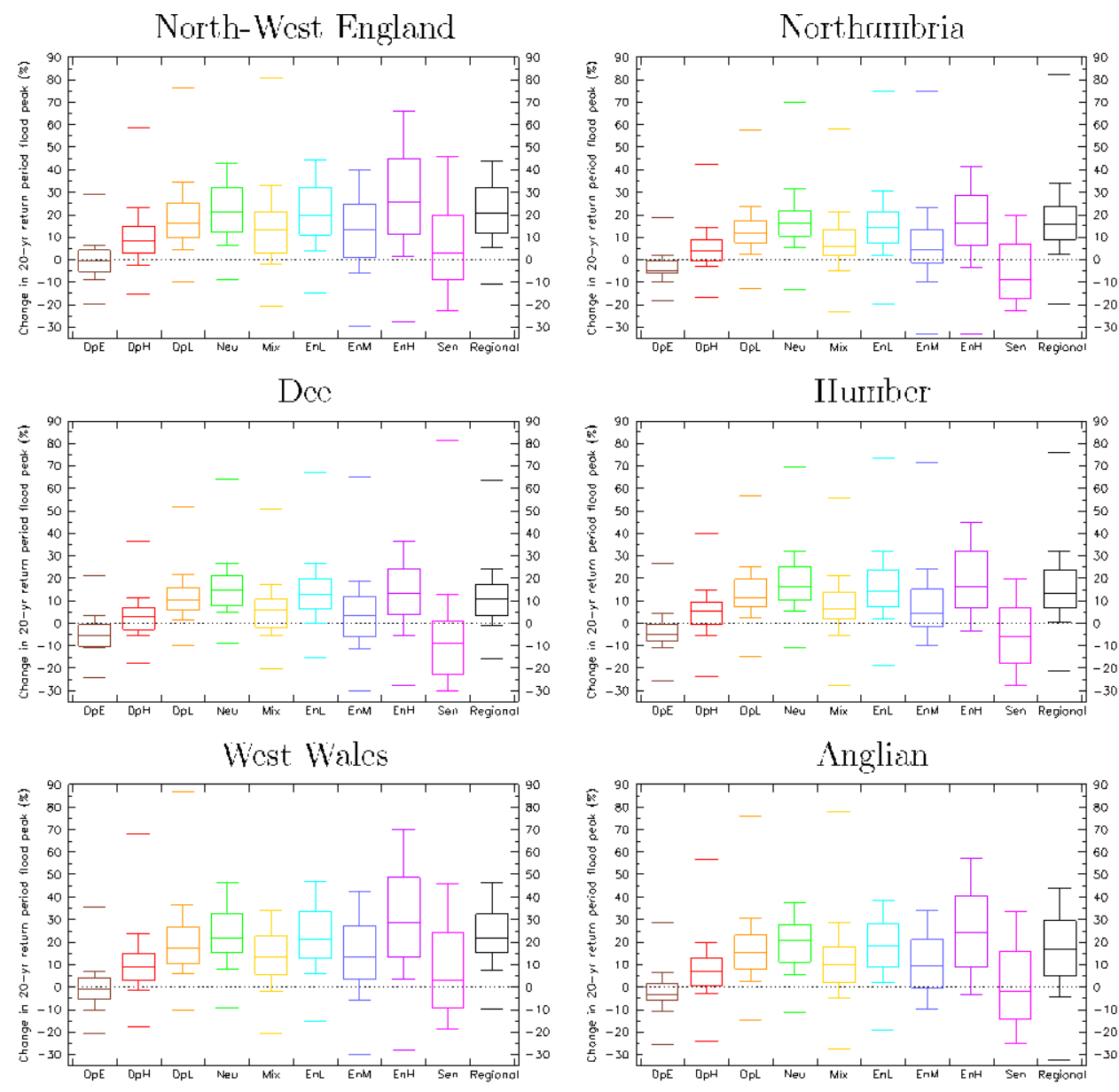

Anglian

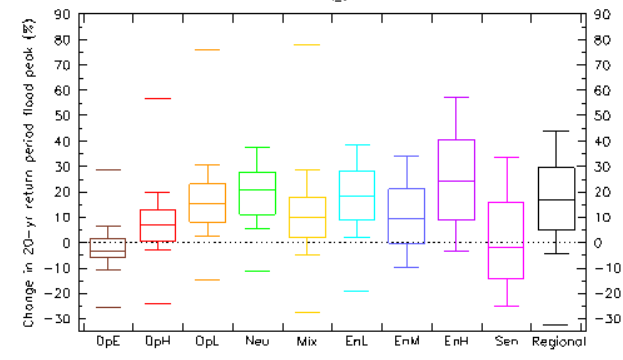

Scrern

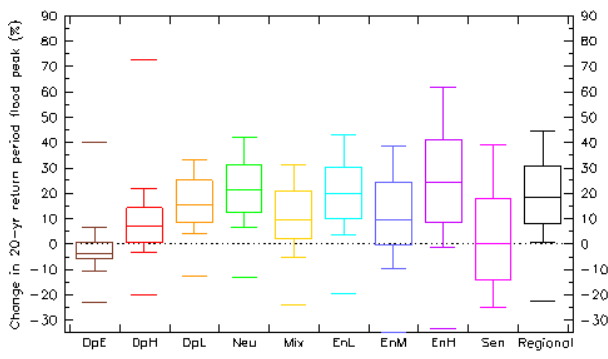

Thamincs

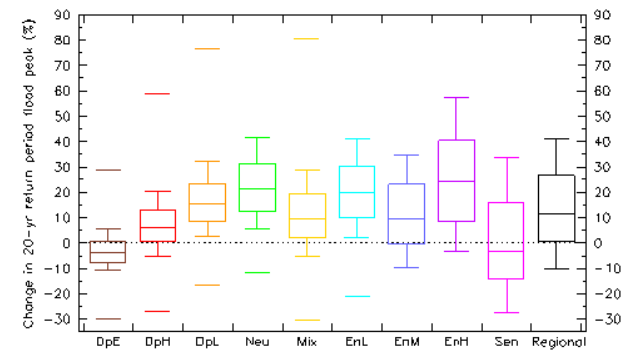

South-West Fngland

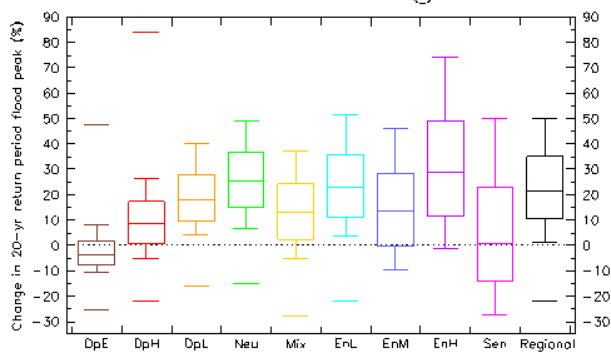

Sonth-Fast Frigland

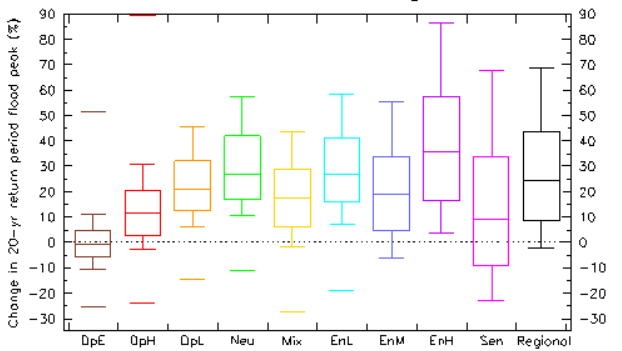

Figure 5 Box-and-whisker plots showing the impact ranges for each response type (DpE - brown, DpH - red, DpL - orange, Neu - green, Mix gold, EnL - cyan, EnM - blue, EnH - purple, Sen - magenta) in each riverbasin region in England and Wales (2080s Medium). Also shown are regional average impact ranges (right-most box-plot for each region; Section 3.3 ). Boxes indicate the $25^{\text {th }}-50^{\text {th }}-75^{\text {th }}$ percentile range; whiskers the $10^{\text {th }}-90^{\text {th }}$ percentile range; additional markers are minima and maxima (if within the plotted range $-35 \%-95 \%$ ). 


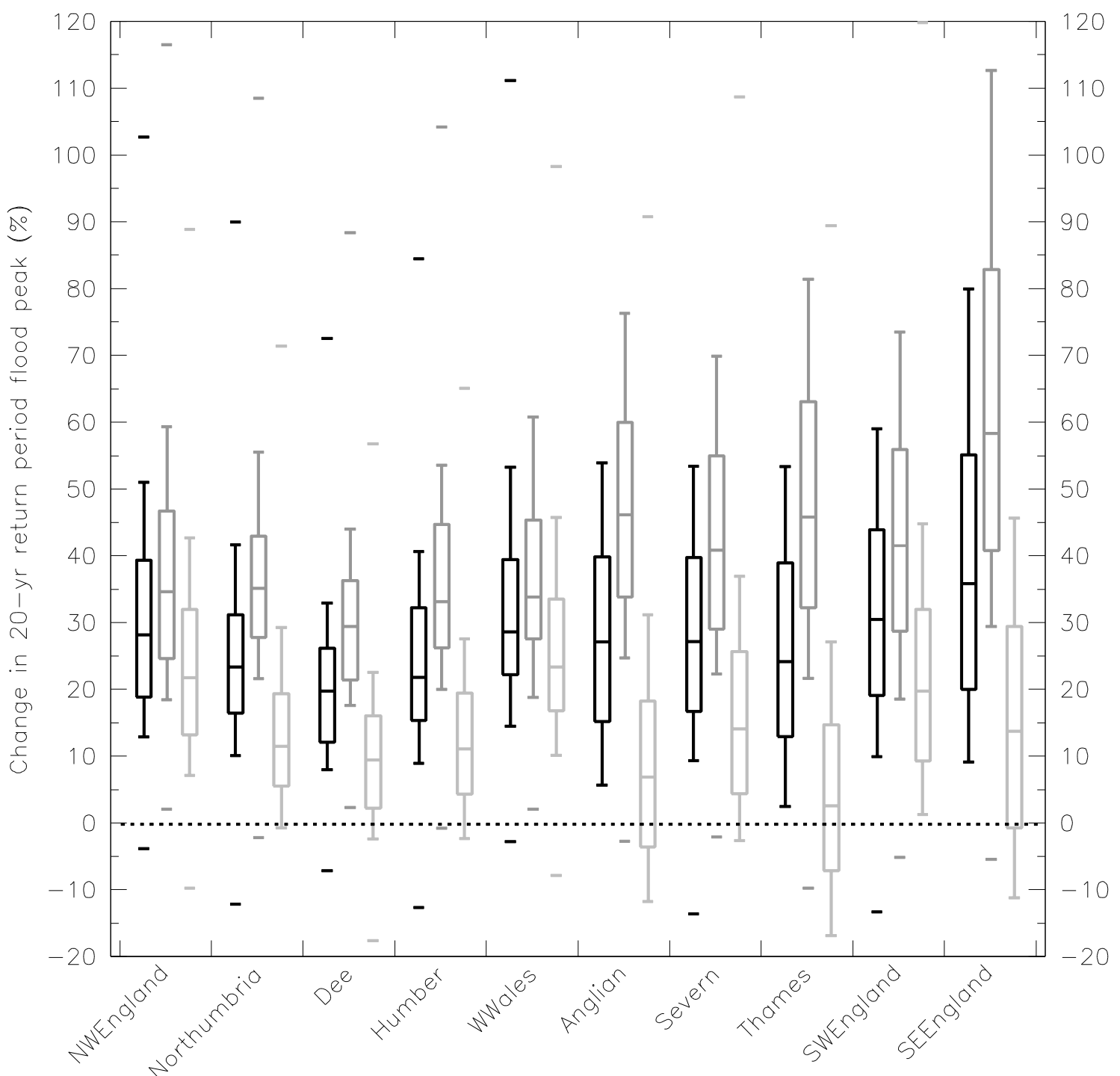

Figure 6 Box-and-whisker plots comparing the central-estimate of the regional average impact ranges (black), including bias correction values (Section 2.4), for each river-basin region in England and Wales (2080s Medium). Additional boxes for each region show alternative ranges when adding \pm 2 sd (mid-grey and light grey respectively). Box-and-whisker percentiles as Figure 5. 


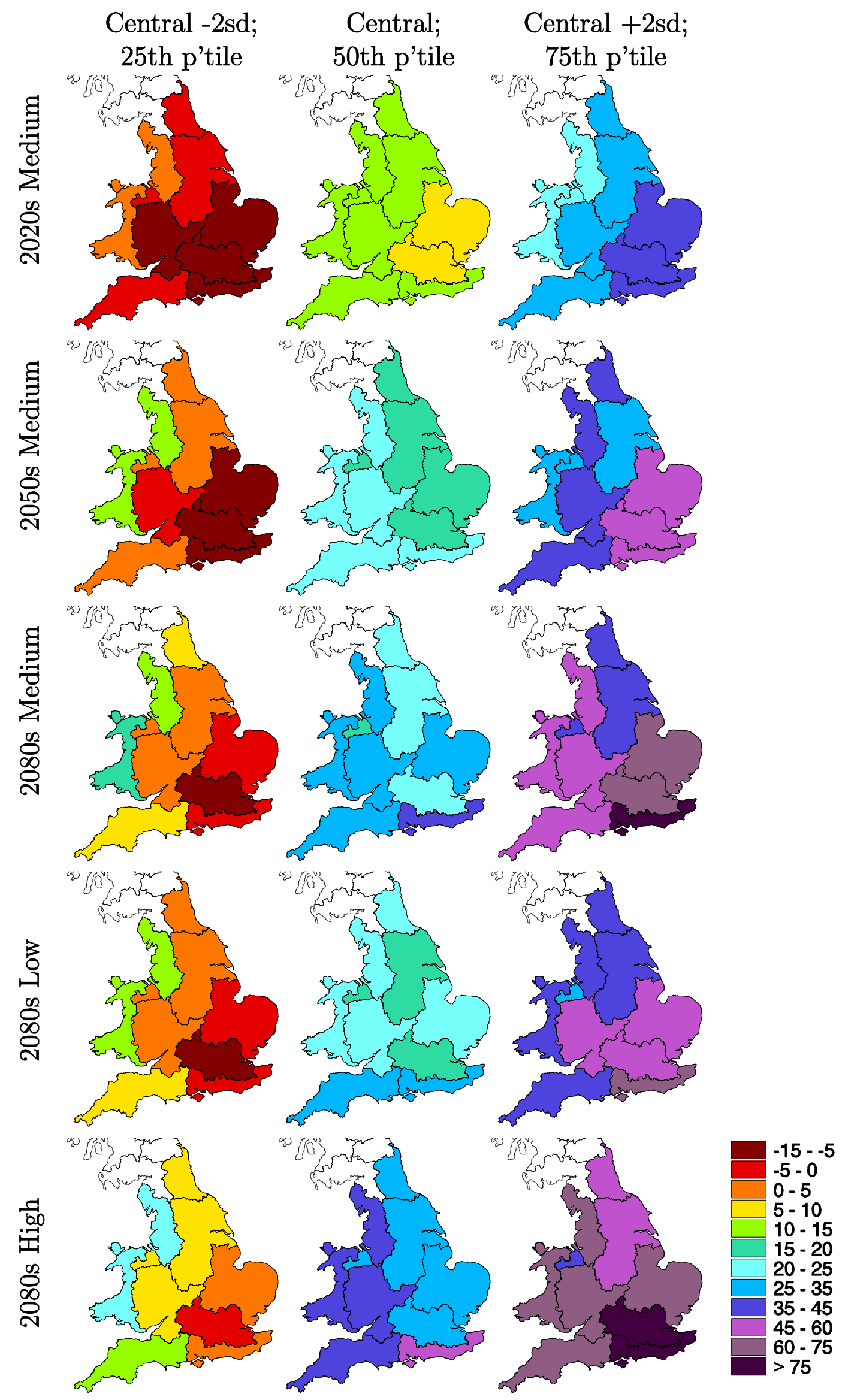

Figure 7 Variation in regional impacts (percentage change in 20-year return period flood peaks) for England and Wales for several timehorizons and emissions scenarios. 